\title{
mRNA compartmentalisation spatially orients tissue morphogenesis
}

${\text { Guilherme } \text { Costa }^{1 *} \text {, Joshua Bradbury }}^{1}$, Nawseen Tarannum ${ }^{1} \&$ Shane P. Herbert ${ }^{1, *}$

${ }^{1}$ Faculty of Biology, Medicine and Health, Michael Smith Building, University of

Manchester, Oxford Road, Manchester, M13 9PT, UK.

*e-mail: guilherme.costa@manchester.ac.uk,shane.herbert@manchester.ac.uk 


\begin{abstract}
Polarised targeting of diverse mRNAs to motile cellular protrusions is a hallmark of cell migration $^{1-3}$. Although a widespread phenomenon, definitive functions for endogenous targeted mRNAs and their relevance to modulation of in-vivo tissue dynamics remain elusive. Here, using single-molecule analysis, endogenous gene-edited mRNAs and zebrafish in-vivo live-cell imaging, we report that mRNA polarisation acts as a molecular compass that orients motile cell polarity and spatially directs tissue movement. Clustering of protrusion-derived RNAseq datasets defined a core $192 \mathrm{bp}$ localisation element underpinning precise mRNA targeting to incipient sites of filopodia formation at cell protrusions. Such targeting of the small GTPase, $R A B 13$, generated tight spatial coupling of mRNA localisation, translation and protein activity, achieving precise subcellular compartmentalisation of RAB13 protein function to create a polarised domain of filopodia extension. Consequently, genomic excision of this localisation element and specific perturbation of endogenous $R A B 13$ targeting - but not translation - depolarised filopodial dynamics in motile endothelial cells and induced miss-patterning of nascent blood vessels in-vivo. Hence, mRNA polarisation, not expression, is the primary spatial determinant of the site of RAB13 action, preventing ectopic functionality at inappropriate subcellular loci and orienting tissue morphogenesis. Considering the unexpected spatial diversity of other polarised mRNA clusters we identified, mRNA-mediated compartmentalisation of protein function at distinct subcellular sites likely coordinates broad aspects of in-vivo tissue behaviour.
\end{abstract}




\section{RESULTS AND DISCUSSION}

Dynamic subcellular polarisation of a myriad of proteins fundamentally shapes the front-rear polarity and directed movement of motile cells ${ }^{4}$. In parallel, cell migration is associated with subcellular polarisation of numerous mRNAs ${ }^{1-3}$. However, precise functional roles for targeted mRNAs remain unclear considering difficulties in identifying and manipulating endogenous transcript $c$ is targeting elements ${ }^{5}$. Likewise, whether this phenomenon is relevant to modulation of tissue dynamics in-vivo remains an open question. To address these issues, we first aimed to define mRNA localisation motifs driving transcript polarisation in motile endothelial cells (ECs), as an initial step towards probing their function in coordinating blood vessel morphogenesis in-vivo. As a starting point, we identified 320 transcripts enriched in fractionated cellular protrusions of migrating primary human umbilical vein ECs (HUVECs) in-vitro (Fig.1a,b, Extended Data Table 1). $k$-means clustering analysis of these data alongside RNAseq datasets from unrelated cell types (NIH/3T3 fibroblasts ${ }^{6}$, MDA-MB231 metastatic breast cancer cells ${ }^{7}$, induced neuronal cells ${ }^{8}$ ) revealed unexpected cell type-specific diversity to transcript polarisation, with just five mRNAs exhibiting universal targeting to protrusions in all cell types tested (cluster $k 5 ;$ RAB13, TRAK2, RASSF3, NET1, KIF1C; Fig.1b, c). Strikingly, cluster $k 5$ mRNAs all shared near-identical spatial distributions by single molecule FISH (smFISH) ${ }^{9}, 10$, being highly polarised to cellular protrusions relative to a control transcript, GAPDH (Fig. 1d-g). Moreover, $k 5$ transcripts were highly spatially distinct from other clusters, such as mRNAs of cluster $k 7$, which exclusively encoded secretory proteins ${ }^{11-13}$ and exhibited less-polarised peri-nuclear targeting (Fig.1g; Extended Data Fig.1b, e-f). Likewise, protrusion localisation of the well-established polarised mRNA, $A C T B^{14}$, was significantly more diffuse than $k 5$ 
mRNAs, as were other cluster $k 2$ members (Fig.1g; Extended Data Fig.1b-d). Finally, protrusion-enriched mRNAs were also tightly clustered according to protein function (Extended Data Fig.1a), with $k 5$ transcripts specifically encoding cell peripheryassociated modulators of vesicle trafficking and membrane remodelling ${ }^{15-19}$. Hence, tight coupling of distinct spatial distributions of mRNAs with discrete protein functionalities indicated that the universal polarisation of cluster $k 5$ mRNAs likely reflected a conserved functional requirement in motile cells.

Considering that the polarisation of cluster $k 5 \mathrm{mRNAs}$ was particularly acute, highly stereotyped and uniquely conserved amongst cell types (Fig.1c-g), we hypothesised that these transcripts employed common targeting mechanisms. The cisregulation of mRNA targeting is widely attributed to localisation elements (LE) within 3' untranslated regions (3'UTR) ${ }^{5,20-24}$. Indeed, despite identification of consensus cisregulatory localisation elements being notoriously difficult, we detected repeat use of a conserved sequence motif in the 3'UTRs of all $k 5$ transcript (Fig.1h; Extended Data Fig.2a). Moreover, this motif was striking in its clustering as five repeats within a short 3'UTR region of $R A B 13$, a known polarised mRNA ${ }^{23,25}$ (Fig. 1h). Potent localisation properties of this region were confirmed upon imaging of exogenous mRNAs in ECs using the MS2 system ${ }^{26}$ (Fig.1i,j; Extended Data Fig.2b). Using this approach, we identified a minimal $192 \mathrm{bp}$ LE encompassing four motif repeats that was both necessary and sufficient to exclusively polarise mRNA at motile EC protrusions (Fig.1i,j). As RAB13 was the only identified RAB small GTPase to exhibit such mRNA polarisation (Fig.1b), we hypothesised that this LE was critical for RAB13 function in motile cells. RAB13 is an established modulator of cortical F-actin crosslinking and cytoskeletal remodelling at leading front of migrating cells, via interaction with its 
effector protein, MICAL-L2 ${ }^{27-29}$. Consistent with this function, live-cell imaging of RAB13 3'UTR dynamics revealed exclusive targeting of mRNA to sites of incipient filopodia formation in cellular protrusions, suggesting tight spatial coupling between $R A B 13$ mRNA localisation and RAB13 protein activity (Fig.2a-c; Supplementary Video1). Furthermore, quantification revealed a putative causal spatial relationship between $R A B 13$ proximity and filopodia frequency/stability (Fig.2b,c). Likewise, induction of cell migration drove a significant increase the levels and polarisation of RAB13 mRNA in-vitro (Extended Data Fig.2c-e), further indicating dynamic involvement in leading-edge remodelling and establishment of cell polarity. Hence, these data revealed that polarisation of $R A B 13$ mRNA may spatially compartmentalise RAB13-mediated F-actin remodelling to orient motile cell polarity.

Studies probing the precise function of endogenous polarised mRNAs in motile cells are currently lacking, predominantly due to difficulties identifying cis targeting motifs and the potential propensity for genomic manipulation of mRNAs to perturb transcript stability and/or translation. However, precise genomic excision of the $R A B 13$ minimal LE in HUVECs using CRISPR-Cas9 tools did not perturb RAB13 protein expression (Extended Data Fig.3a-e), but did eradicate the polarised spatial pattern of $R A B 13$ localisation, such that transcript became diffusely distributed in ECs similar to GAPDH (Fig. 2e-g). Importantly, this loss of RAB13 mRNA polarisation - but not loss of expression - was sufficient to depolarise filopodial dynamics in motile ECs (Fig.2hk; Extended Data Fig.3f). When co-cultured with fibroblasts to mimic polarised blood vessel sprouting ${ }^{30}$, Wild type (Wt) HUVECs exhibited highly polarised filopodia extensions biased towards the leading edge of motile cell protrusions (Fig.2j). In contrast, filopodia in HUVECs lacking the RAB13 LE were no longer spatially 
compartmentalised and were ectopically distributed homogeneously along the distalproximal axis of migrating cells (Fig. $2 \mathrm{j}$ ). As a consequence, mutant ECs exhibited a significant increase in overall filopodia frequency (Fig.2i). Hence, this work revealed that tight control of $R A B 13$ mRNA localisation spatially specifies a polarised domain of filopodia extension in motile cells (Fig.2k).

These striking observations suggested that $R A B 13$ mRNA polarisation acts to exclusively spatially compartmentalise RAB13-mediated filopodia extension at distal sites. As such, targeting of $R A B 13$ mRNA and local translation could effectively block ectopic protein function at inappropriate subcellular loci to orient motile cells. However, this could only be achieved if the sites of $R A B 13$ mRNA localisation, translation and protein function were all tightly spatially coupled. Indeed, such coupling may be consistent with longstading proposals that newly translated RABs form a discrete protein pool from mature RABs, potentially with distinct interaction partners (e.g. specific RAB escorting proteins and GDP dissociation inhibitors) and separate biological functions ${ }^{31-33}$. Hence, local translation of polarised $R A B 13$ transcript may generate nascent protein with a distinct functionality to mature RAB13 at specific subcellular sites, thus achieving tight spatial compartmentalisation of RAB13-mediated membrane remodelling. As predicted, such coupling of polarised $R A B 13 \mathrm{mRNA}$ localisation with local translation was confirmed in EC protrusions upon detection of nascent protein using puromycinilation-proximity ligation assays (Puro-PLA) ${ }^{34}$. HUVECs were cultured on Transwells and, prior to short incubation with puromycin, cell bodies removed to exclude detection of nascent puromycin-labelled proteins transported from the cell body to protrusions (Fig.3a). Isolated EC protrusions readily incorporated puromycin, which could be blocked upon pre-incubated with the 
translation inhibitor anisomycin (Extended Data Fig.4a), indicating active protein translation at the leading edge of migrating ECs. Importantly, Puro-PLA on isolated EC protrusions using antibodies recognising incorporated puromycin and endogenous RAB13 detected numerous distinct punctate of nascent synthesised RAB13, unlike anisomycin pre-treated and antibody-free controls (Fig.3a-c; Extended Data Fig.4b,c). Hence, polarised targeting of $R A B 13$ mRNA to motile cell protrusions drives local RAB13 translation. Moreover, spatial control of mRNA polarisation and local translation was coupled to regional compartmentalisation of RAB13 protein function, as loss of endogenous $R A B 13$ specifically disrupted filopodia dynamics only at sites of mRNA targeting (Fig.3e-g; Extended Data Fig.4d). Knockdown did not perturb RAB13independent filopodia at proximal regions in ECs, but significantly depleted filopodia numbers at distal sites. This was not simply a consequence of spatial targeting of mature protein, as immunofluorescence assays revealed that RAB13 was homogeneously distributed throughout migrating cells (Extended Data Fig.4e). Alternatively, it was the location of $R A B 13$ mRNA itself that defined the domain of RAB13-dependent filopodia dynamics, as excision of the LE and diffuse miss-localisation of $R A B 13$ mRNA was sufficient to drive ectopic depolarised filopodia (Fig.2h-k). Hence, the site of $R A B 13$ mRNA localisation, translation and protein function are tightly spatially coupled in migrating cells. Thus, polarisation of $R A B 13$ transcript forms a molecular compass that achieves precise subcellular compartmentalisation of protein function, defines a polarised domain of filopodia extension and ultimately orients motile cell polarity (Fig.3g).

Although a widespread phenomenon, the functional role for localised mRNAs in tissue migration and vertebrate morphogenesis remains unexplored. Hence, having 
defined a key role for mRNA polarisation in the spatial control of EC behaviour invitro, we then sought to define the broader relevance of this phenomenon to modulation of tissue dynamics in-vivo. The production of polarised filopodial protrusions is a characteristic hallmark of motile endothelial tip cells, which lead new blood vessel branches during angiogenesis ${ }^{35,36}$. As such, using live-cell imaging approaches in the zebrafish model system, we probed the function of rab13 mRNA polarisation in the control of tip cell behaviour and angiogenesis in-vivo. Firstly, we generated a novel vascular-specific MCP-GFPnls transgenic strain, Tg(flilep:MCP-GFPnls), and monitored the dynamics of rab13 3'UTR mRNA targeting during intersegmental vessel (ISV) angiogenesis ${ }^{36}$ (Fig.4a; Extended Data Fig.5a and Supplementary Video2). Dynamic accumulation of MCP-GFPnls adjacent to and within filopodia at the leading edge of ISV tip cells revealed that rab13 mRNA targeting in-vivo closely mirrored the 3'UTR-driven polarisation of RAB13 mRNA in-vitro. Similarly, CRISPR-Cas9mediated excision of a genomic fragment of the endogenous rab13 3'UTR locus ( $\triangle 3^{\prime}$ UTR) confirmed that it also contained LEs critical for polarisation of zebrafish rab13 mRNA (Fig.4b,c; Extended Data Fig.5b-e). In particular, smFISH for zebrafish rabl3 in explanted cells from dissociated $\mathrm{rab} 13^{\triangle 3^{\prime} U T R / \triangle 3^{\prime} U T R}$ mutant embryos confirmed that mRNAs lacking these LEs were more diffusely distributed (Fig.4c), similar to observations in human ECs (Fig.2f). Hence, we reveal a previously unappreciated and conserved role for 3'UTR LEs in the dynamic polarisation of mRNA during cell migration in-vivo.

During ISV branching, migrating tip cells must make key directional decisions, particularly when negotiating the multi-tissue junction of the horizontal myoseptum ${ }^{37-39}$ (HM; Extended Data Fig.5f). Hence, we hypothesised that mRNA localisation-mediated 
orientation of EC filopodia may indeed generate spatial cues that direct vascular tissue movement. Consistent with a key role for mRNA polarisation in the spatial coordination of vascular morphogenesis, live-cell imaging of ISVs branching in Wt and $r a b 13^{\triangle 3^{\prime} U T R / \triangle 3^{\prime} U T R}$ embryos revealed that loss of $r a b 13$ polarisation severely perturbed tip cell path-finding decisions (Fig.4d,e). Unlike ISVs in Wt and $r a b 13^{+/ \Delta 3^{\prime} U T R}$ embryos that efficiently negotiated their way past the HM position, ISVs in $r a b 13^{\triangle 3^{\prime} U T R / \triangle 3^{\prime} U T R}$ mutants struggled with this directional decision, resulting in a 7-fold increase in tip cells exhibiting ectopic miss-directed branches (Fig.4d,e). Importantly, rab13 mRNA stability was unperturbed in $r a b 13^{\triangle 3^{\prime} U T R / \triangle 3^{\prime} U T R}$ mutant embryos (Extended Data Fig.5g), consistent with excision of the RAB13 LE in-vitro (Fig.2e), confirming that observed defects were not due to decreased rab13 expression but were a consequence of perturbed mRNA localisation. Thus, we provide the first in-vivo evidence that spatial targeting of mRNAs and precise compartmentalisation of protein function generates key directional cues that orient motile cells and drive tissue morphogenesis (Fig.4f).

Here, using precise gene-editing of endogenous mRNAs, we reveal that tight spatial coupling of mRNA localisation, translation and protein function achieves the exclusive subcellular compartmentalisation of protein function. Hence, we define a novel paradigm for the spatial control of motile cell polarity and oriented tissue movement in-vivo. Taking into account that $R A B 13$ is one of only five mRNAs exhibiting conserved polarisation in all cell types tested, this function is likely a universally conserved mechanism for spatial coordination of complex morphogenetic events. Moreover, it is striking that all five cluster $k 5$ mRNAs encode highly dynamic membrane trafficking and/or small GTPase-regulating proteins, all known to modulate cell motility. Hence, for classes of proteins normally constant in motion, mRNA- 
polarisation may be essential to spatially compartmentalise and precisely fix their site of function. Finally, this work reveals an unexpected spatial diversity to other identified clusters of polarised mRNAs. Considering our observations that the sites of mRNA targeting and protein function are tightly coupled, this raises the exciting possibility that other distinct mRNA distributions, such as the perinuclear localisation of cluster $k 7$, reflect even broader functionalities for compartmentalised gene expression/function in the coordination of diverse aspects of tissue development and disease. 


\section{REFERENCES}

1. Bovaird, S., Patel, D., Padilla, J.A. \& Lecuyer, E. Biological functions, regulatory mechanisms, and disease relevance of RNA localization pathways. FEBS letters 592, 2948-2972 (2018).

2. Buxbaum, A.R., Haimovich, G. \& Singer, R.H. In the right place at the right time: visualizing and understanding mRNA localization. Nature reviews. Molecular cell biology 16, 95-109 (2015).

3. Liao, G., Mingle, L., Van De Water, L. \& Liu, G. Control of cell migration through mRNA localization and local translation. Wiley interdisciplinary reviews. RNA 6, 1-15 (2015).

4. Mayor, R. \& Etienne-Manneville, S. The front and rear of collective cell migration. Nature reviews. Molecular cell biology 17, 97-109 (2016).

5. Jambhekar, A. \& Derisi, J.L. Cis-acting determinants of asymmetric, cytoplasmic RNA transport. Rna 13, 625-642 (2007).

6. Wang, T., Hamilla, S., Cam, M., Aranda-Espinoza, H. \& Mili, S. Extracellular matrix stiffness and cell contractility control RNA localization to promote cell migration. Nature communications 8, 896 (2017).

7. Mardakheh, F.K. et al. Global Analysis of mRNA, Translation, and Protein Localization: Local Translation Is a Key Regulator of Cell Protrusions. Developmental cell 35, 344-357 (2015).

8. Zappulo, A. et al. RNA localization is a key determinant of neurite-enriched proteome. Nature communications $\mathbf{8 ,} 583$ (2017).

9. Tsanov, N. et al. smiFISH and FISH-quant - a flexible single RNA detection approach with super-resolution capability. Nucleic acids research 44, e165 (2016).

10. Raj, A., van den Bogaard, P., Rifkin, S.A., van Oudenaarden, A. \& Tyagi, S. Imaging individual mRNA molecules using multiple singly labeled probes. Nature methods 5, 877-879 (2008).

11. Bradham, D.M., Igarashi, A., Potter, R.L. \& Grotendorst, G.R. Connective tissue growth factor: a cysteine-rich mitogen secreted by human vascular endothelial cells is related to the SRC-induced immediate early gene product CEF-10. J Cell Biol 114, 1285-1294 (1991).

12. O'Brien, T.P., Yang, G.P., Sanders, L. \& Lau, L.F. Expression of cyr61, a growth factor-inducible immediate-early gene. Molecular and cellular biology 10, 3569-3577 (1990).

13. Yanagisawa, M., Kurihara, H., Kimura, S., Goto, K. \& Masaki, T. A novel peptide vasoconstrictor, endothelin, is produced by vascular endothelium and 
modulates smooth muscle Ca2+ channels. Journal of hypertension. Supplement: official journal of the International Society of Hypertension 6, S188-191 (1988).

14. Condeelis, J. \& Singer, R.H. How and why does beta-actin mRNA target? Biology of the cell 97, 97-110 (2005).

15. Srougi, M.C. \& Burridge, K. The nuclear guanine nucleotide exchange factors Ect 2 and Net1 regulate RhoB-mediated cell death after DNA damage. PloS one 6, e17108 (2011).

16. Wu, C. et al. Rab13-dependent trafficking of RhoA is required for directional migration and angiogenesis. J Biol Chem 286, 23511-23520 (2011).

17. Kopp, P. et al. The kinesin KIF1C and microtubule plus ends regulate podosome dynamics in macrophages. Molecular biology of the cell 17, 2811-2823 (2006).

18. Brickley, K., Smith, M.J., Beck, M. \& Stephenson, F.A. GRIF-1 and OIP106, members of a novel gene family of coiled-coil domain proteins: association in vivo and in vitro with kinesin. J Biol Chem 280, 14723-14732 (2005).

19. Tommasi, S. et al. RASSF3 and NORE1: identification and cloning of two human homologues of the putative tumor suppressor gene RASSF1. Oncogene 21, 2713-2720 (2002).

20. Hotz, M. \& Nelson, W.J. Pumilio-dependent localization of mRNAs at the cell front coordinates multiple pathways required for chemotaxis. Nature communications 8, 1366 (2017).

21. Maizels, Y. et al. Localization of cofilin mRNA to the leading edge of migrating cells promotes directed cell migration. Journal of cell science 128, 1922-1933 (2015).

22. Liao, G., Simone, B. \& Liu, G. Mis-localization of Arp2 mRNA impairs persistence of directional cell migration. Experimental cell research 317, 812$822(2011)$.

23. Mili, S., Moissoglu, K. \& Macara, I.G. Genome-wide screen reveals APCassociated RNAs enriched in cell protrusions. Nature 453, 115-119 (2008).

24. Kislauskis, E.H., Zhu, X. \& Singer, R.H. Sequences responsible for intracellular localization of beta-actin messenger RNA also affect cell phenotype. J Cell Biol 127, 441-451 (1994).

25. Jakobsen, K.R. et al. Direct RNA sequencing mediated identification of mRNA localized in protrusions of human MDA-MB-231 metastatic breast cancer cells. Journal of molecular signaling 8, 9 (2013).

26. Bertrand, E. et al. Localization of ASH1 mRNA particles in living yeast. Molecular cell 2, 437-445 (1998). 
27. Ioannou, M.S. et al. DENND2B activates Rab13 at the leading edge of migrating cells and promotes metastatic behavior. J Cell Biol 208, 629-648 (2015).

28. Sakane, A. et al. Junctional Rab13-binding protein (JRAB) regulates cell spreading via filamins. Genes Cells 18, 810-822 (2013).

29. Sakane, A. et al. Rab13 small G protein and junctional Rab13-binding protein (JRAB) orchestrate actin cytoskeletal organization during epithelial junctional development. J Biol Chem 287, 42455-42468 (2012).

30. Hetheridge, C., Mavria, G. \& Mellor, H. Uses of the in vitro endothelialfibroblast organotypic co-culture assay in angiogenesis research. Biochemical Society transactions 39, 1597-1600 (2011).

31. Seabra, M.C., Mules, E.H. \& Hume, A.N. Rab GTPases, intracellular traffic and disease. Trends Mol Med 8, 23-30 (2002).

32. Shen, F. \& Seabra, M.C. Mechanism of digeranylgeranylation of Rab proteins. Formation of a complex between monogeranylgeranyl-Rab and Rab escort protein. J Biol Chem 271, $3692-3698$ (1996).

33. Pfeffer, S.R., Dirac-Svejstrup, A.B. \& Soldati, T. Rab GDP dissociation inhibitor: putting rab GTPases in the right place. J Biol Chem 270, 17057-17059 (1995).

34. tom Dieck, S. et al. Direct visualization of newly synthesized target proteins in situ. Nature methods 12, 411-414 (2015).

35. Gerhardt, H. et al. VEGF guides angiogenic sprouting utilizing endothelial tip cell filopodia. J Cell Biol 161, 1163-1177 (2003).

36. Isogai, S., Lawson, N.D., Torrealday, S., Horiguchi, M. \& Weinstein, B.M. Angiogenic network formation in the developing vertebrate trunk. Development 130, 5281-5290 (2003).

37. Lamont, R.E., Lamont, E.J. \& Childs, S.J. Antagonistic interactions among Plexins regulate the timing of intersegmental vessel formation. Developmental biology 331, 199-209 (2009).

38. Lu, X. et al. The netrin receptor UNC5B mediates guidance events controlling morphogenesis of the vascular system. Nature 432, 179-186 (2004).

39. Torres-Vazquez, J. et al. Semaphorin-plexin signaling guides patterning of the developing vasculature. Developmental cell 7, 117-123 (2004). 


\section{METHODS}

\section{Zebrafish husbandry}

Zebrafish were grown and maintained according to UK Home Office regulation guidelines and all studies were approved by the University of Manchester Ethical Review Board.

\section{Embryo micro-injections and generation of zebrafish strains}

To generate the transgenic zebrafish strain Tg(flilep:MCP-GFPnls) using Tol2 transposon transgenesis, 32 pg of Cerulean-H2B:basflilep:MCP-GFPnls Tol2-based plasmid was co-injected with $32 \mathrm{pg}$ Tol2 mRNA into one-cell stage AB zebrafish embryos. The next day, embryos with mosaic GFP expression were selected, raised into adulthood and then outbred to $\mathrm{AB}$ zebrafish to identify founders with germ-line transmission of the transgene. Adult Tg(flilep:MCP-GFPnls) were inbred and one-cell stage embryos were co-injected with 32 pg of Cerulean-H2B:basflilep:Lyn-mCherry24xMS2-rab13-3'UTR Tol2-based plasmid and 32 pg Tol2 mRNA for mosaic expression analysis.

The mutant rab13 $\Delta 3$ 'UTR strain was generated with CRISPR-Cas9 tools. One-cell stage $\operatorname{Tg}(k d r l: E G F P)^{s 843}$ embryos $^{40}$ were injected with $150 \mathrm{pg}$ of each in vitro transcribed gRNA and co-injected with 150 pg Cas9 NLS nuclease (New England Biolabs). Embryos were raised to adulthood and outbred to AB zebrafish to identify founders with germ-line transmission deletions in the rab13 3'UTR. Heterozygous animals harbouring a 482-nucleotide deletion in the rab13 3'UTR $\left(\operatorname{Tg}(k d r l: E G F P)^{s 843}\right.$

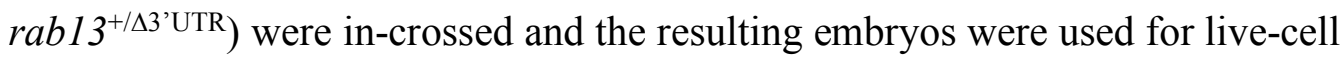
imaging analysis. 


\section{gRNA generation and in vitro transcription}

The online CRISPRscan tool ${ }^{41}$ was used to design gRNAs targeting the zebrafish 3'UTR region in the rab13 locus (Extended Data Table 2). Next, $0.3 \mu \mathrm{M}$ oligonucleotides comprising the target sequences (flanked by the $\mathrm{T} 7$ promoter and the Tail annealing sequence) were mixed with $0.45 \mu \mathrm{M}$ Tail primer (Extended Data Table 2) and PCR amplified with Platinum Pfx DNA polymerase (ThermoFisher Scientific) in a T100 thermal cycler (BioRad). The following cycling conditions were used: 1 cycle of initial denaturation at $94{ }^{\circ} \mathrm{C}$ for 10 minutes, 30 cycles of denaturation at $94{ }^{\circ} \mathrm{C}$ for 30 seconds, annealing at $45^{\circ} \mathrm{C}$ for 30 seconds, extension at $68^{\circ} \mathrm{C}$ for 30 seconds, and a final extension cycle at $68{ }^{\circ} \mathrm{C}$ for 7 minutes. Subsequently, $200 \mathrm{ng}$ of PCR amplified templates were used to transcribe gRNAs using a MEGAshortscript T7 Transcription Kit (ThermoFisher Scientific), following the manufacturer's recommendations. To synthesise Tol 2 mRNA, $1 \mu \mathrm{g}$ NotI-linearised pCS2-TP plasmid was transcribed using a SP6 mMESSAGE mMACHINE kit (ThermoFisher Scientific) according to manufacturer's protocol.

\section{Embryo genotyping}

Genomic DNA was extracted by incubating either whole embryos or embryo heads in lysis buffer (10 mM Tris $\mathrm{HCl}$ pH8, 1 mM EDTA, $80 \mathrm{mM} \mathrm{KCl,} 0.3 \%$ NP40, $0.3 \%$ Tween) containing $0.5 \mu \mathrm{g} / \mu 1$ Proteinase $\mathrm{K}$ (Promega) at $55^{\circ} \mathrm{C}$ for $1-2$ hours, followed by a denaturation step at $95{ }^{\circ} \mathrm{C}$ for 15 minutes in a T100 thermal cycler. Genotyping PCR was performed using $2 \mu 1$ genomic DNA, $0.4 \mu \mathrm{M}$ zebrafish genotyping primers (Extended Data Fig.5A, Table 2) and 1 x MyTaq Red DNA Polymerase (Bioline) 
according to the manufacturer's protocol in a T100 thermal cycler. PCR reactions were resolved in $1 \%$ agarose (Bioline) gels containing $0.5 \mu \mathrm{g} / \mathrm{ml}$ Ethidium Bromide (Sigma) for analysis. PCR products were cloned into TOPO-TA vectors (ThermoFisher Scientific) according to the manufacturer's protocol and analysed via Sanger sequencing on an ABI 3730 device.

\section{Cell culture, scratch wound and co-culture angiogenesis assays}

Trunks of 26-28 hpf embryos were incubated in Trypsin-EDTA solution (Sigma) at 28 ${ }^{\circ} \mathrm{C}$ for 15 minutes. Trypsinisation was quenched with complete L-15 medium (Sigma) containing $10 \%$ Fetal Bovine Serum (FBS, Sigma) and $10 \mathrm{U} / \mathrm{ml}-100 \mu \mathrm{g} / \mathrm{ml}$ PenicillinStreptomycin (Sigma). Cells were pelleted at $2000 \mathrm{rpm}$ for 5 minutes at room temperature (RT), resuspended in complete L-15 medium, plated on Laminin-coated (Sigma) coverslips in 24 well plates and maintained at $28^{\circ} \mathrm{C}$ for 18 hours. HUVECs (PromoCell) were cultured in complete ECGM2 (PromoCell) in gelatincoated (Millipore) dishes. Human Pulmonary Fibroblasts (HPF; PromoCell) were cultured in M199 (ThermoFisher Scientific) containing $10 \%$ FBS, $50 \mu \mathrm{g} / \mathrm{ml}$ Gentamycin (Sigma) and 50 ng/ml Amphotericin (Sigma). Brain endothelial (bEnd.3) were cultured in DMEM (Sigma) supplemented with $10 \%$ FBS, $10 \mathrm{ng} / \mathrm{ml}$ Recombinant Human VEGF-A (PeproTech) and 10 U/ml-100 $\mu \mathrm{g} / \mathrm{ml}$ Penicillin-Streptomycin.

For scratch wound assays, HUVECs cultured on gelatin coated coverslips were grown to confluence and used in scratch wound assays as described elsewhere ${ }^{42}$. Co-cultures of HUVEC and HPF and the corresponding siRNA-mediated knockdown experiments were performed as previously described by Hetheridge et al. ${ }^{30}$. 


\section{CRISPR-Cas9 cell editing and cell transfections}

To edit RAB13 locus, HUVECs were transfected with Alt-R CRISPR-Cas9

ribonucleoprotein complexes (Integrated DNA Technologies) targeting the 90-282nt localisation element within the 3'UTR. Briefly, each sequence-specific crRNA (Extended Data Table 2) was mixed with tracrRNA at 1:1 $50 \mu \mathrm{M}$, incubated at $95{ }^{\circ} \mathrm{C}$ for 5 minutes in a T100 thermal cycler and allowed to cool to RT for 60 minutes. Next, $12 \mu \mathrm{M}$ each crRNA:tracrRNA (gRNA) was incubated with $20 \mu \mathrm{M}$ Alt-R Cas9 nuclease in PBS (Sigma) at RT for 20 minutes to form ribonucleoprotein complexes and mixed with 500x1033 HUVECs. Additionally, $2 \mu \mathrm{g}$ pmaxGFP Vector (Lonza) was included in the HUVEC-ribonucleoprotein mix to identify transfected cells. Transfections were performed in a Nucleofector 2b Device (Lonza), using a HUVEC Nucleofector kit (Lonza) according to manufacturer's instructions and the cells were further cultured for 72 hours. Afterwards, single GFP-expressing cells were isolated in a FACS Aria Fusion cell sorter (BD Biosciences) into gelatin-coated 96 well plates to grow individual clones. Genomic DNA was extracted from expanded HUVEC clones and PCR-analysed with sequence-specific primers (Extended Data Fig.3A, Table 2) as described for zebrafish embryo genotyping.

Knockdown experiments were performed with ON-TARGETplus Non-targeting control or $R A B 13$ siRNAs (Horizon) using GeneFECTOR (VennNova) as previously described $^{30}$.

For in vitro MS2 experiments, bEnd.3 cells were transfected with pcDNA3-LynmCherry, pCS2-MCP-GFPnls and different versions of pcDNA3-HBB-24XMS2SLRAB 13 3'UTR. Briefly, 100x $10^{3}$ cells / well cultured in 6 well plates were transfected 
with $0.8 \mu \mathrm{g}$ each plasmid DNA using Lipofectamin2000 following the manufacturer's protocol (ThermoFisher Scientific) and analysed 48 hours later.

\section{Transwell assays and cell body / protrusion fractionation}

Transwell experiments to segregate cell bodies and protrusions were performed as described elsewhere ${ }^{23}$, with the following modifications: $1.5 \times 10^{6}$ HUVECs were cultured for 2 hours in $24 \mathrm{~mm}$ Transwells (Costar), containing $3 \mu \mathrm{m}$-pore polycarbonate membranes, in M199 (ThermoFisher Scientific) supplemented with $1 \%$ FBS. Subsequently, 25ng/ml VEGF-A was added to the lower chambers to promote cell migration over the next hour. While only 1 Transwell was used for the cell body fraction, 2 Transwells were used to harvest each HUVEC protrusion sample.

\section{RNA isolation, qPCR and RNAseq}

Embryo and cell-derived RNA was isolated using a RNAqueous-Micro kit (ThermoFisher Scientific) according to the manufacturer's protocol. For gene expression analysis, cDNA was synthesised with a High-Capacity RNA-to-cDNA kit (ThermoFisher Scientific) following the manufacturer's protocol. qPCR experiments were performed with 1-2 $\mu \mathrm{l}$ cDNA, $0.25 \mu \mathrm{M}$ gene-specific primers (Extended Data Table 2) and 1 x Power SYBR Green Master Mix (ThermoFisher Scientific) in a StepOne Real-Time PCR System (Applied Biosystems). GAPDH expression was used to normalise gene expression levels and the relative mRNA levels were analysed with the $2^{-\Delta \Delta C T}$ method.

For RNAseq, quality and integrity of RNA samples obtained from HUVEC cell bodies and protrusions were assessed using a 2200 TapeStation (Agilent Technologies). Next, 
RNAseq libraries were generated using the TruSeq Stranded mRNA assay (Illumina) according to the manufacturer's protocol. Adapter indices were used to multiplex libraries, which were pooled prior to cluster generation using a cBot instrument. The loaded flow-cell was then paired-end sequenced $(76+76$ cycles, plus indices $)$ on an Illumina HiSeq4000 instrument. Finally, the output data was demultiplexed (allowing one mismatch) and BCL-to-Fastq conversion performed using Illumina's bcl2fastq software, version 2.17.1.14.

\section{SmFISH}

Zebrafish cells and HUVECs cultured on either Laminin or gelatin-coated coverslips, respectively, were fixed in methanol free $4 \%$ formaldehyde (ThermoFisher Scientific) and used in smFISH assays. Briefly, cells were permeabilised with $70 \%$ Ethanol at RT for 1 hour or $4{ }^{\circ} \mathrm{C}$ overnight, washed with smFISH wash buffer (2 X SSC, $10 \%$ formamide) and incubated with smFISH probes (Extended Data Table 3) in smFISH hybridisation buffer ( $10 \%$ dextran sulfate, $2 \mathrm{X} \mathrm{SSC}, 10 \%$ formamide) at $37^{\circ} \mathrm{C}$ overnight. Afterwards, cells were washed with smFISH wash buffer twice at $37^{\circ} \mathrm{C}$ for 30 minutes, washed once with 2 X SSC for 10 minutes, counterstaining with $1 \mu \mathrm{g} / \mathrm{ml}$ DAPI (Sigma) and washed twice with PBS for 5 minutes at RT. Coverslips were airdried and mounted on microscope slides with ProLong Gold Antifade Mountant (ThermoFisher Scientific). All probes targeting protrusion-enriched mRNAs were designed with Stellaris Probe Designer (LGC Biosearch Technologies), synthesised and labelled with Quasar 570 (LGC Biosearch Technologies) or Alexa 594 fluorophores (Integrated DNA Technologies). Co-hybridisation experiments were carried out with predesigned GAPDH probes labelled with Quasar 670 (LGC Biosearch Technologies). 


\section{Puro-PLA and immunofluorescence (IF)}

For Puro-PLA, cell bodies of HUVECs cultured in Transwells were scraped off and remaining protrusions were exposed to $3 \mu \mathrm{M}$ Puromycin (Sigma) added to lower chambers for 6 minutes. In translation inhibition experiments, $40 \mu \mathrm{M}$ Anisomycin (Sigma) was added to the lower Transwell chamber 30 minutes before cell body removal and 6 minutes after cell body removal together with $3 \mu \mathrm{M}$ Puromycin. Subsequently, HUVEC protrusions grown in Transwell membranes were fixed in methanol free $4 \%$ formaldehyde, removed from the Transwell inserts and used in PuroPLA experiments as described elsewhere ${ }^{34}$. Following the Puro-PLA protocol, Transwell membranes were incubated for 20 minutes with 1:40 Alexa Fluor 488 Phalloidin (ThermoFisher Scientific) in PBS, washed in Duolink wash buffer B (Sigma) and mounted on microscope slides with Duolink In Situ Mounting Medium containing DAPI (Sigma).

For IF experiments, cells and Transwell membranes containing protrusions were permeabilised in PBS containing 0.2-0.5 \% Triton-X100 (Sigma), blocked in $4 \%$ goat serum (Sigma) for 15 minutes, and incubated with primary antibodies in blocking solution at $4{ }^{\circ} \mathrm{C}$ overnight. Next, cells were washed in PBS containing $0.2 \%$ Tween, incubated with secondary antibodies at RT for 1 hour, counterstaining with $1 \mu \mathrm{g} / \mathrm{ml}$ DAPI and washed again. Transwell membranes were further incubated with 1:40 Phalloidin Alexa Fluor 488 (ThermoFisher Scientific) in PBS at RT for 20 minutes before washing. Cells and Transwell membranes were mounted with ProLong Gold Antifade Mountant (ThermoFisher Scientific). 


\section{Western blotting}

Proteins were extracted with RIPA buffer $(25 \mathrm{mM}$ Tris- $\mathrm{HCl} \mathrm{pH}$ 7.6, $150 \mathrm{mM} \mathrm{NaCl}, 1 \%$ NP-40, $1 \%$ sodium deoxycholate, and $0.1 \%$ SDS) and quantified with Pierce BCA protein assay kit (ThermoFisher Scientific) following the supplier's recommendations. Samples were denatured with Laemmli buffer $(250 \mathrm{mM}$ Tris-HCl pH 6.8, $2 \%$ SDS, 10 $\%$ glycerol, $0.0025 \%$ bromophenol blue, $2.5 \% \beta$-mercaptoethanol) at $95{ }^{\circ} \mathrm{C}$ for 5 minutes, loaded on 10\% Mini-PROTEAN TGX precast protein gels (Bio-Rad) and separated in a Mini-PROTEAN Electrophoresis System (Bio-Rad). Proteins were transferred onto nitrocellulose membranes using a Trans-Blot Turbo Transfer System RTA kit following the manufacturer's protocols (Bio-Rad). Subsequently, membranes were blocked in $5 \%$ milk (Sigma) or $5 \%$ BSA (Sigma) in TBS containing $0.1 \%$ Tween at RT for 1 hour and incubated with primary antibodies at $4{ }^{\circ} \mathrm{C}$ overnight. The next day membranes were washed with TBS containing $0.1 \%$ Tween, incubated with secondary antibodies at RT for 1 hour and washed again. Signal detection was carried out with SuperSignal West Dura Extended Duration Substrate (ThermoFisher Scientific) according to the supplier's recommendations.

\section{Antibodies}

Primary and secondary antibodies were used at the following concentrations: 1:1600 mouse PECAM-1 89C2 (Cell Signaling Technology), 1:100 rabbit RAB13 (Puro-PLA and IF, Millipore), 1:1000 rabbit RAB13 (Western blotting, Cambridge Bioscience), 1:3500 mouse Puromycin (Kerafast), 1:1000 rabbit $\beta$-Tubulin 9F3 (Cell Signaling Technology), 1:200 mouse ZO-1 1A12 (ThermoFisher Scientific), 1:500 goat anti- 
mouse Alexa Fluor 488 or Alexa Fluor 568, (ThermoFisher Scientific) and 1:5000 goat anti-mouse HRP-linked antibody (Cell Signaling Technology).

\section{Plasmid construction}

The pCS2-MCP-GFPnls plasmid used in in vitro MS2-system assays was generated excising a MCP-GFPnls fragment with SpeI and KpnI from pMS2-GFP, a gift from Robert Singer (Addgene plasmid \# 27121) ${ }^{43}$, and subcloning it into a pCS2+ vector using the XbaI and KpnI sites.

To construct the Cerulean-H2B:basflilep:MCP-GFPnls Tol2-based plasmid for in vivo studies, MCP-GFPnls was amplified from pMS2-GFP with sequence $0.3 \mu \mathrm{M}$ specific primers (Extended Data Table 2) and Platinum Pfx DNA polymerase in a T100 thermal cycler. Subsequently, the PCR product was cloned into a pDONR221 P3-P2 using Gateway Technology (ThermoFisher Scientific) according to manufacturer's manual. The final Tol2-based construct was assembled into the pTol2Dest(R1R2) (Addgene plasmid \# 73484) ${ }^{44}$ using Gateway 3-fragment recombination with pE(L1L4)Cerulean$\mathrm{H} 2 \mathrm{~B}$ in the first position, $\mathrm{pE}(\mathrm{R} 4 \mathrm{R} 3)$ basflilep $^{7}$ in the second position and $\mathrm{pE}(\mathrm{L} 3 \mathrm{~L} 2) \mathrm{MCP}-\mathrm{GFPnl}$ in the third position.

For in vitro MS2-system experiments, the 3'UTR of human RAB13 was PCR amplified with $0.3 \mu \mathrm{M}$ sequence-specific primers (Extended Data Table 2) using Platinum Pfx DNA polymerase in a T100 thermal cycler and the resulting PCR product was cloned using a Zero Blunt PCR cloning kit (ThermoFisher Scientific), following the manufacturer's manual. Next, the human $H B B$ gene was PCR amplified using $0.3 \mu \mathrm{M}$ sequence-specific primers (Extended Data Table 2) and Platinum Pfx DNA polymerase in a T100 thermal cycler and cloned into the NotI and BamHI sites of the pCR4- 
24XMS2SL-stable plasmid, a gift from Robert Singer (Addgene plasmid \# 31865) ${ }^{26}$. Subsequently, a multiple cloning site (MCS, Extended Data Table 2) was introduced into the BglII and SpeI sites of pCR4- $H B B-24 \mathrm{XMS} 2 \mathrm{SL}$ and the recombinant $H B B$ 24XMS2SL-MCS sequence was subcloned into the pcDNA3 mammalian expression vector (ThermoFisher Scientific) using the NotI and XbaI sites. The full-length 482nt $R A B 13$ 3'UTR was then sub-cloned into pcDNA3-HBB-24XMS2SL-MCS using NheI and XhoI sites. Alternatively, truncated and deletion versions of RAB13 3'UTR were generated by PCR using $0.3 \mu \mathrm{M}$ sequence-specific primers (Extended Data Table 2) and Platinum Pfx DNA polymerase or using QuikChange II Site-Directed Mutagenesis kit (Agilent Technologies) following the manufacturer's instructions and introduced into the pcDNA3- $H B B-24 \mathrm{XMS} 2 \mathrm{SL}-\mathrm{MCS}$ using the NheI and XhoI sites.

In order to generate the zebrafish MS2-system reporter construct, the 24XMS2SL cassette was firstly subcloned from pCR4-24XMS2SL-stable into a $k d r l: L y n-m C h e r r y$ Tol2 based plasmid ${ }^{45}$ using a BamHI site. Next, the zebrafish rab13 3'UTR was PCR amplified with $0.4 \mu \mathrm{M}$ sequence specific primers (Extended Data Table 2) and MyTaq Red DNA Polymerase from zebrafish genomic DNA in a T100 thermal cycler and then subcloned into the Tol2 kdrl:Lyn-mCherry-24XMS2SL plasmid using NheI and BgIII sites. The resulting Lyn-mCherry-24XMS2SL-rab13 3'UTR recombinant sequence was amplified with $0.3 \mu \mathrm{M}$ sequence-specific primers (Extended Data Table 2) and Platinum Pfx DNA polymerase in a T100 thermal cycler and subcloned into a pDONR221 P3-P2 using Gateway Technology. Lastly, the final Tol2-based construct was assembled into the pTol2Dest(R1R2) using Gateway 3-fragment recombination with $\mathrm{pE}(\mathrm{L} 1 \mathrm{~L} 4)$ Cerulean-H2B in the first position, $\mathrm{pE}(\mathrm{R} 4 \mathrm{R} 3)$ basflilep in the second position and Lyn-mCherry-24XMS2SL-rab13 3'UTR in the third position. 
All plasmid maps and details are available upon request.

\section{Microscopy}

Confocal time-lapse imaging of zebrafish embryos was carried out as previously described $^{45}$. MS2 system-transfected cells were live imaged every 5 seconds in a Nikon A1R inverted confocal microscope equipped with an Okolab incubation chamber, using a $60 \mathrm{X}$ objective. Fixed images of cultured cells and Transwell membranes were acquired on an Olympus IX83 inverted microscope using Lumencor LED excitation, either a $60 \mathrm{X} / 1.42$ PlanApo or a $100 \mathrm{X} / 1.35$ UplanApo objective and a Sedat QUAD (DAPI/FITC/TRITC/Cy5) filter set (Chroma 89000). The images were collected using a R6 (Qimaging) CCD camera with a Z optical spacing of $0.2 \mu \mathrm{m}$. Raw images were then deconvolved using the Huygens Pro software (SVI) and maximum intensity projections of these images were used for analysis.

\section{smFISH spot quantification, Polarisation Index (PI) and filopodia analysis}

Processed smFISH images were used to calculate mRNA polarisation with the PI metric developed by Park et al. ${ }^{46}$ and to assess mRNA spot number with FISHQuant ${ }^{47}$. For the studies of filopodia distance to GFP signal in MS2-system experiments, filopodia parameters (position, duration, and frequency) of MS2-system transfected cells were determined using Filopodyan plugin for FIJI ${ }^{48}$. Subsequently, the coordinates of GFP particles were extracted with the TrackMate plugin for FIJI ${ }^{49}$ and the Euclidean distance between the base of newly formed filopodia and the nearest GFP particle was calculated. 


\section{Statistics}

All data are represented as mean \pm standard deviation. Statistical analysis of the data was carried out using GraphPad prism software. Differences in smFISH spot, Puro-PLA and filopodia numbers, protein and RNA levels, ISV length and branching were interrogated using t-tests when the data were normally distributed or Mann-Whitney tests when the data did not pass normality tests. The correlation between GFP distance and filopodia frequency or duration were assessed with Spearman correlation tests. Statistical significance is reported for $P<0.05$. $k$-means clustering of RNAseq data was performed using Morpheus (Broad Institute, https://software.broadinstitute.org/morpheus/index.html). The number of clusters was defined by the number of cell types and by the possible transcript statuses (enriched or depleted) $-2^{3}=8$. Gene Ontology studies were performed using DAVID ${ }^{50,51}$. 


\section{METHODS REFERENCES}

40. Jin, S.W., Beis, D., Mitchell, T., Chen, J.N. \& Stainier, D.Y. Cellular and molecular analyses of vascular tube and lumen formation in zebrafish. Development 132, 5199-5209 (2005).

41. Moreno-Mateos, M.A. et al. CRISPRscan: designing highly efficient sgRNAs for CRISPR-Cas9 targeting in vivo. Nature methods 12, 982-988 (2015).

42. Liang, C.C., Park, A.Y. \& Guan, J.L. In vitro scratch assay: a convenient and inexpensive method for analysis of cell migration in vitro. Nature protocols $\mathbf{2}$, 329-333 (2007).

43. Fusco, D. et al. Single mRNA molecules demonstrate probabilistic movement in living mammalian cells. Curr Biol 13, 161-167 (2003).

44. Villefranc, J.A., Amigo, J. \& Lawson, N.D. Gateway compatible vectors for analysis of gene function in the zebrafish. Developmental dynamics : an official publication of the American Association of Anatomists 236, 3077-3087 (2007).

45. Costa, G. et al. Asymmetric division coordinates collective cell migration in angiogenesis. Nat Cell Biol 18, 1292-1301 (2016).

46. Park, H.Y., Trcek, T., Wells, A.L., Chao, J.A. \& Singer, R.H. An unbiased analysis method to quantify mRNA localization reveals its correlation with cell motility. Cell Rep 1, 179-184 (2012).

47. Mueller, F. et al. FISH-quant: automatic counting of transcripts in 3D FISH images. Nature methods 10, 277-278 (2013).

48. Urbancic, V. et al. Filopodyan: An open-source pipeline for the analysis of filopodia. J Cell Biol 216, 3405-3422 (2017).

49. Tinevez, J.Y. et al. TrackMate: An open and extensible platform for singleparticle tracking. Methods 115, 80-90 (2017).

50. Huang da, W., Sherman, B.T. \& Lempicki, R.A. Bioinformatics enrichment tools: paths toward the comprehensive functional analysis of large gene lists. Nucleic acids research 37, 1-13 (2009).

51. Huang da, W., Sherman, B.T. \& Lempicki, R.A. Systematic and integrative analysis of large gene lists using DAVID bioinformatics resources. Nature protocols 4, 44-57 (2009). 


\section{ACKNOWLEDGEMENTS}

We wish to thank members of the University of Manchester Biological Services, Genomic Technologies, Bioimaging Facilities and Flow Cytometry Facilities, for technical support. We are grateful to E. Schuman and S. tom Dieck at the MPI for Brain Research, Frankfurt, Germany, for help and guidance setting up Puro-PLA assays. We also thank N. Papalopulu and group members at the University of Manchester, UK, for critical feedback, reagents and materials. This work was funded by the Wellcome Trust (095718/Z/11/Z to S.P.H.), Wellcome Institutional Strategic Support Fund (7064646 to G.C.) and the British Heart Foundation (PG/16/2/31863 to S.P.H.).

\section{AUTHOR CONTRIBUTIONS}

Conceptualisation, G.C. and S.P.H.; Methodology, G.C.; Formal Analysis, G.C.; Investigation, G.C., J.B. and N.T.; Writing - Original Draft, G.C. and S.P.H.; Supervision, G.C. and S.P.H.; Funding acquisition, G.C. and S.P.H.

\section{COMPETING INTERESTS}

The authors declare no competing interests

\section{ADDITIONAL INFORMATION}

Correspondence and requests for materials should be addressed to S.P.H. or G.C. 


\section{FIGURE LEGENDS}

Figure 1. Clustering of RNAseq datasets defines a 192 bp localisation element driving mRNA targeting to protrusions. a, Strategy used in the RNAseq screen of mRNAs enriched in motile protrusions of HUVECs migrating through Transwell membranes. b, RNAseq data are plotted in reads per kilobase of transcripts per million reads (RPKM) against fold change (FC) levels of protrusions over cell bodies; lightgrey data are transcripts with low expression and not considered; data are mean values represented in $\log _{2}(\mathrm{n}=2$ replicates). c, Heat map represents the $k$ means clustering of transcript FC levels (protrusions over cell bodies) of other published extracted RNAseq datasets. The corresponding HUVEC FC levels are shown in parallel. d, smFISH codetection of $k 5$ mRNAs and GAPDH in exemplar HUVECs. e, Polarisation Index (PI) of $k 5$ mRNAs and GAPDH in co-detected in HUVECs ( $\mathrm{n} \geq 28$ cells each cohybridization). f, $k 5$ mRNAs PIs plotted against respective GAPDH PIs. The slope of the coloured lines represents the average $k 5 \mathrm{mRNA} / G A P D H$ PI ratio. g, Comparison of the mRNA distribution pattern of transcripts clustered in $k 2, k 5, k 7$ and $G A P D H$. $\mathbf{h}$, Diagram of $k 5$ mRNA 3'UTRs and the positions of the RNA motif shared between transcripts. i, Representative bEnd.3 cells co-transfected with plasmids expressing LynmCherry, MCP-GFPnls and 24xMS2-RAB13 3'UTR or 24xMS2. Arrowhead: nonnuclear localisation of MCP-GFPnls. $\mathbf{j}$, Percentage of bEND. 3 cells with MCP-GFP localised to protrusions when co-transfected with deletion versions of RAB13 3'UTR tagged with $24 x$ MS2 hairpins; ( $\mathrm{n} \geq 10$ cells each triplicate). Data are mean \pm s.d. $* * P<0.01, * * * P<0.001, * * * * P<0.0001$. Arrows: orientation of RNA localisation. Scale bars: $20 \mu \mathrm{m}$. 


\section{Figure 2. $R A B 13$ mRNA polarisation spatially orients filopodia dynamics. a,}

Representative time-lapse microscopy of a bEnd.3 cell co-transfected with plasmids expressing Lyn-mCherry, MCP-GFPnls and 24xMS2-RAB13 3'UTR. b, Frequency of newly formed filopodia formed within $5 \mu \mathrm{m}$ intervals relative to the MCP-GFPnls signal ( $\mathrm{n}=98$ filopodia from 4 replicates). c, Distance to MCP-GFPnls signal of newly formed filopodia plotted against their duration. d, CRISPR-Cas9 strategy to excise the localisation element (LE) in the RAB13 3'UTR of HUVECs. e, smFISH co-detection of $R A B 13$ and control GAPDH in exemplar wild type (Wt) and $\triangle \mathrm{LE}$ HUVECs. Arrows: orientation of RNA localisation. f, Polarisation Index (PI) of RAB13 and GAPDH codetected in Wt and $\triangle$ LE HUVECs ( $\mathrm{n}=29$ cells each genotype). g, RAB13 PI plotted against respective $G A P D H$ PI. The line slopes represent the average $R A B 13 / G A P D H$ PI ratio. h, Exemplar Wt and $\triangle$ LE HUVECs co-cultured on fibroblast monolayers. $\mathbf{i}$ and $\mathbf{j}$, Number of filopodia detected in co-cultured HUVECs per $\mu \mathrm{m}$ (i) and within $12 \mu \mathrm{m}$ intervals relative to cell distal tip (j). k. Illustration of the spatial relationship between $R A B 13$ mRNA localisation and sites of filipodia production. Data are mean \pm s.d. ${ }^{*} P<0.05,{ }^{* *} P<0.01, P^{* * *}<0.001$, ns: not significant $(P>0.05)$. Arrowheads: filopodia. Scale bars: $6 \mu \mathrm{m}(\mathbf{h}) ; 10 \mu \mathrm{m}(\mathbf{a}) ; 20 \mu \mathrm{m}(\mathbf{e})$. 
Figure 3. mRNA polarisation achieves spatial compartmentalisation of RAB13 translation and protein function. a, Strategy used to detect local protein synthesis in protrusions formed by HUVECs migrating through Transwell membranes. b, Representative Puro-PLA experiments detecting newly synthesised RAB13 in HUVEC protrusions present in the lower side of Transwell membranes. Arrowheads: Puro-PLA punctae. c, Quantification of RAB13 Puro-PLA punctae normalised to protrusion area; ( $\mathrm{n} \geq 20$ protrusions each duplicate). d, Exemplar control (ctrl) and $R A B 13$ siRNA-treated HUVECs co-cultured on fibroblast monolayers. e and $\mathbf{f}$, Number of filopodia detected in co-cultured HUVECs per $\mu \mathrm{m}$ (e) and within $12 \mu \mathrm{m}$ intervals relative to cell distal tip (f). $\mathbf{g}$, Illustration of the spatial relationship between the sites of $R A B 13 \mathrm{mRNA}$ localisation, local translation and RAB13 protein-mediated filopodia distribution. Data are mean \pm s.d. $* * P<0.01, * * * P<0.001, * * * * P<0.0001$, ns: not significant $(P>0.05)$. Scale bars: $10 \mu \mathrm{m}$. 
Figure 4. rab13 mRNA polarisation orients blood vessel morphogenesis. a, Timelapse microscopy of a representative $T g$ (flilep:MCP-GFPnls) $\left(\mathrm{T}_{0}=28\right.$ hours post fertilisation (hpf)) ISV cell displaying mosaic expression of rab13 3'UTR tagged with Lyn-mCherry and 24xMS2 hairpins. Arrowheads: non-nuclear localisation of MCPGFPnls. Arrows: direction of ISV sprouting. b, smFISH detection of rabl3 mRNA in 3'UTR $+/+$ and $\Delta / \Delta$ cultured zebrafish embryo cells. c, Polarisation Index of rab13 detected by smFISH in individual zebrafish cells ( $\mathrm{n} \geq 33$ cells each genotype). $\mathbf{d}$, Timelapse confocal microscopy of representative rab13 3'UTR $+/+$ and $\Delta / \Delta$ $\operatorname{Tg}(k d r l: E G F P)^{s 843}$ embryos starting at $25 \mathrm{hpf}\left(\mathrm{T}_{0}\right)$. Arrowheads: extra branches emerging from the main ISVs at the HM position. e, Frequency of ISV ectopic branching occurring at the HM ( $\mathrm{n} \geq 35$ ISVs each genotype). f, Illustration of the role for $R A B 13$ mRNA localisation, local translation and compartmentalisation of RAB13 function in defining the orientation of EC filopodia dynamics, motile EC polarity and blood vessel pathfinding. $P^{*}<0.05,{ }^{* *} P<0.01$. Data are mean \pm s.d. Scale bars: $10 \mu \mathrm{m}$ (b), $50 \mu \mathrm{m}$ (a and d). 
Figure 1

a



HUVECs in $1 \%$ FCS

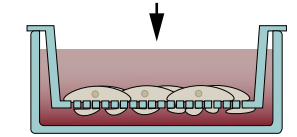

+ VEGF-A in lower chamber

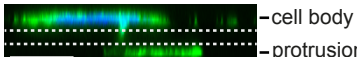



d
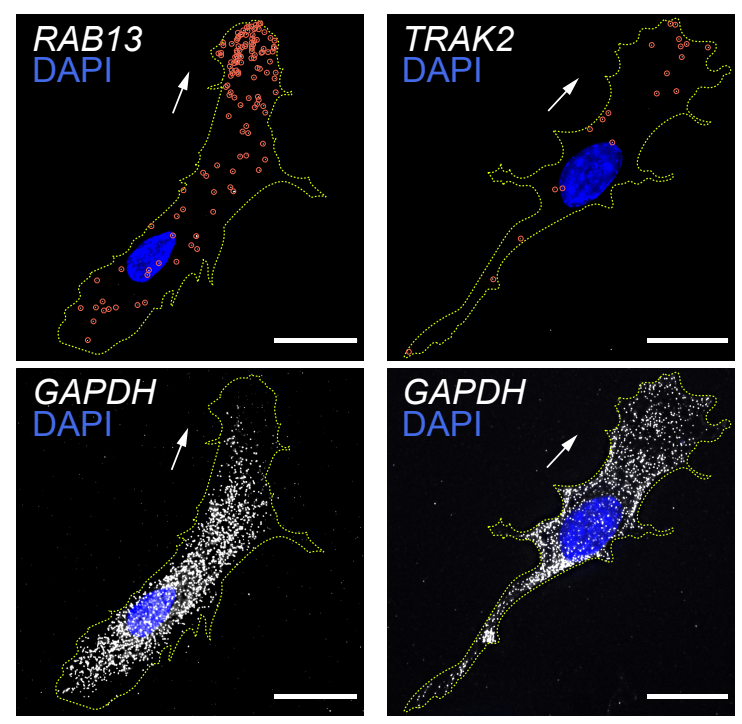

e

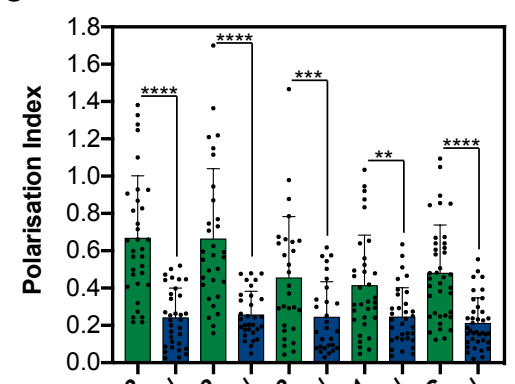

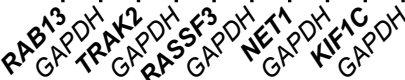
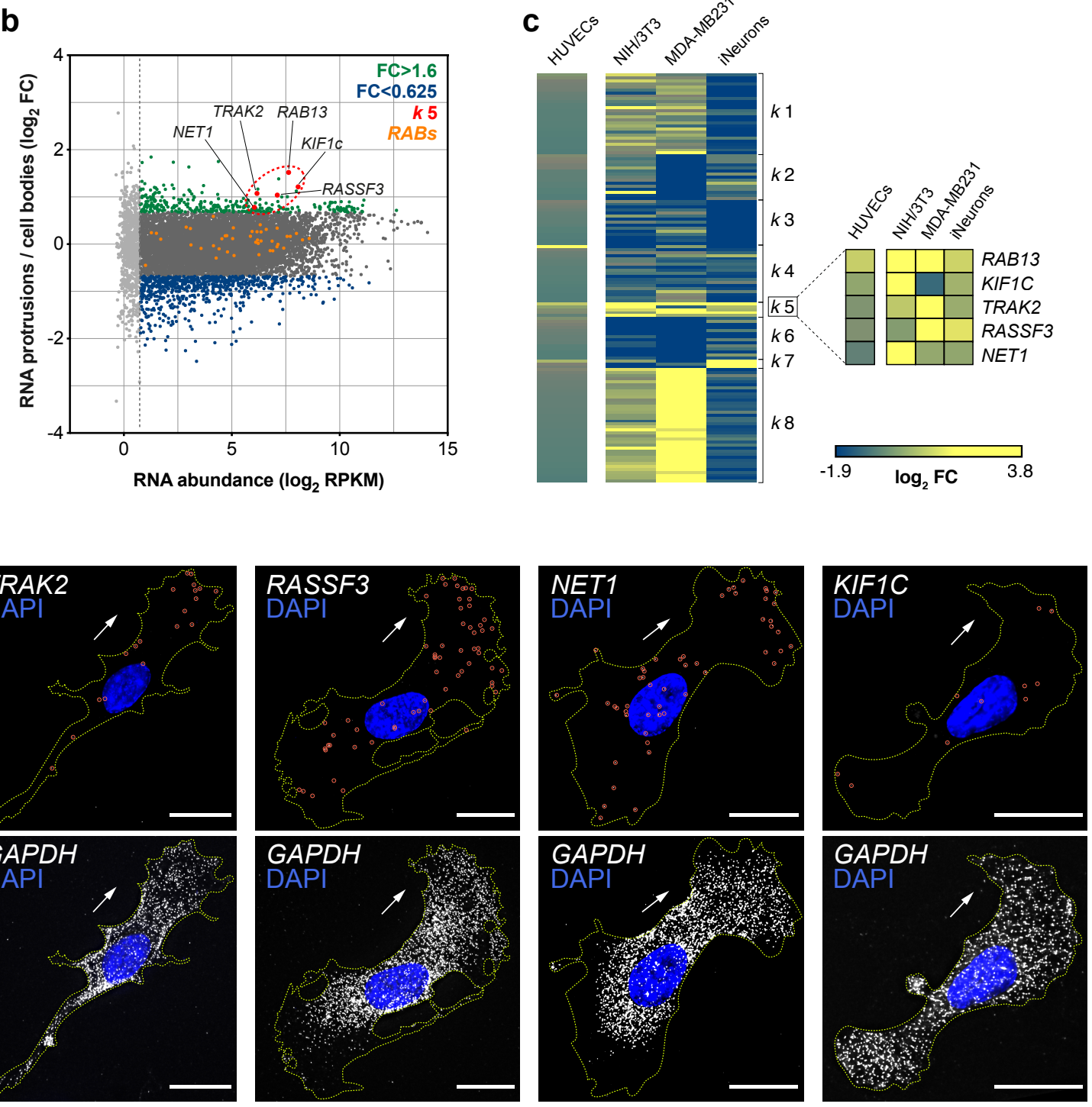

f

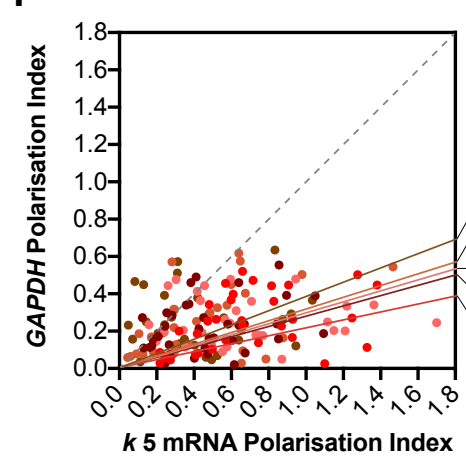

g

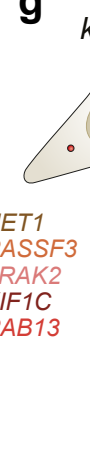

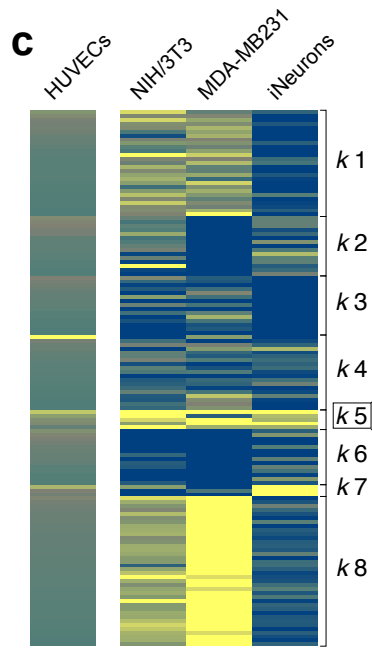
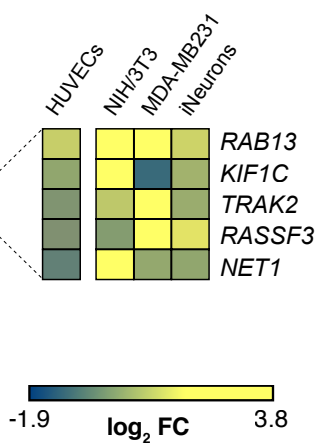

i
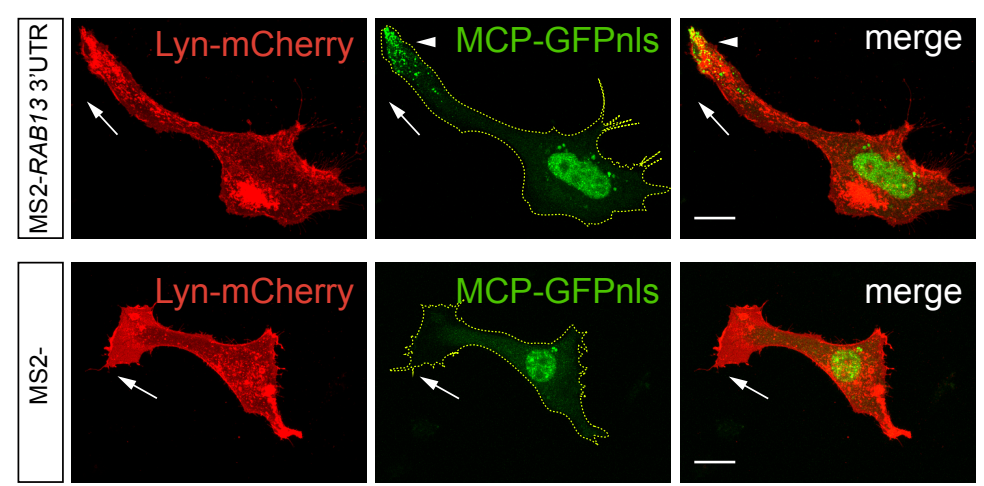

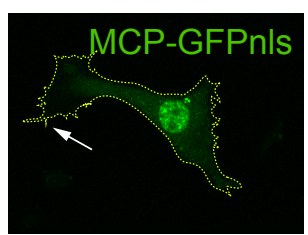



j

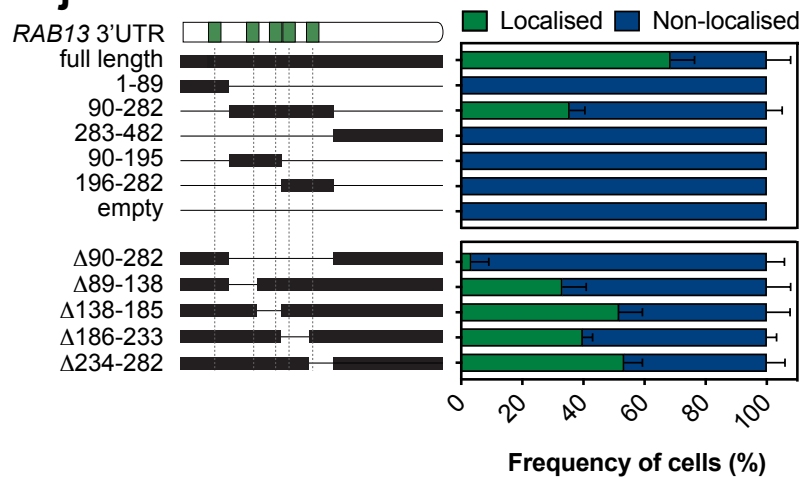


bioRxiv preprint doi: https://doi.org/10.1101/374850; this version posted August 11, 2019. The copyright holder for this preprint (which was not certified by peer review) is the author/funder. All rights reserved. No reuse allowed without permission.

Figure 2

a

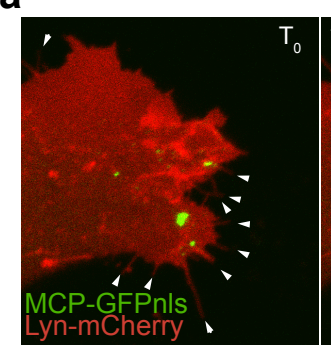

b

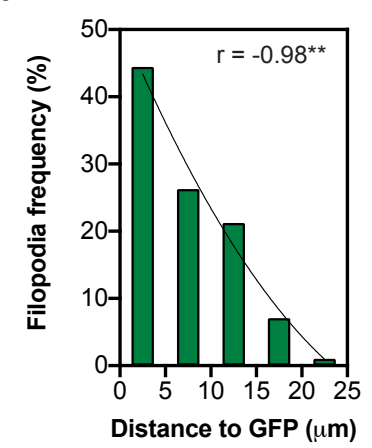

e
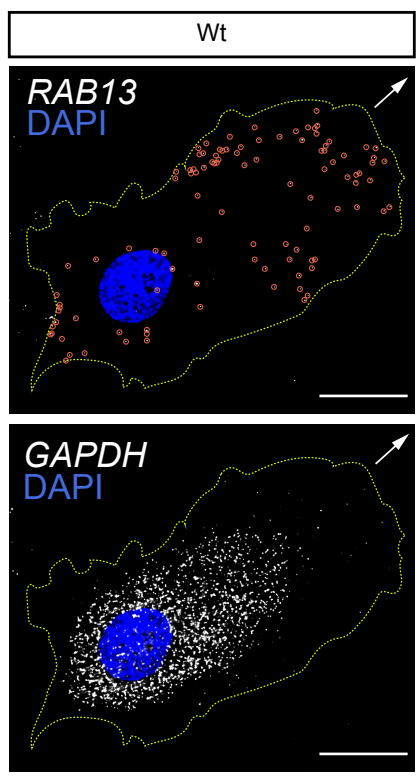

f

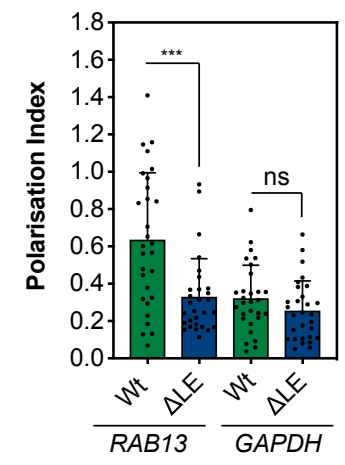

g
C


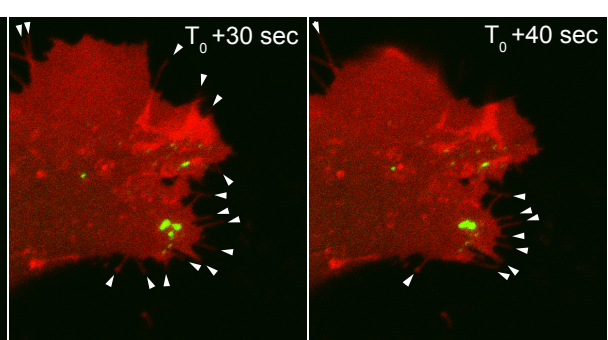

d

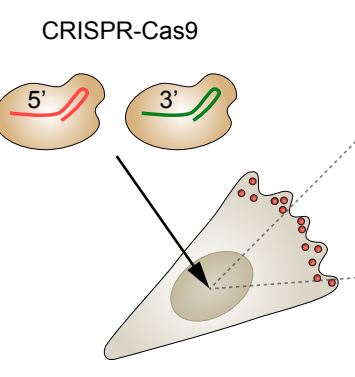

RAB13 3'UTR

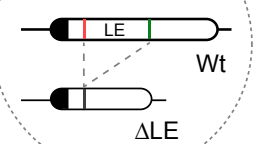

h
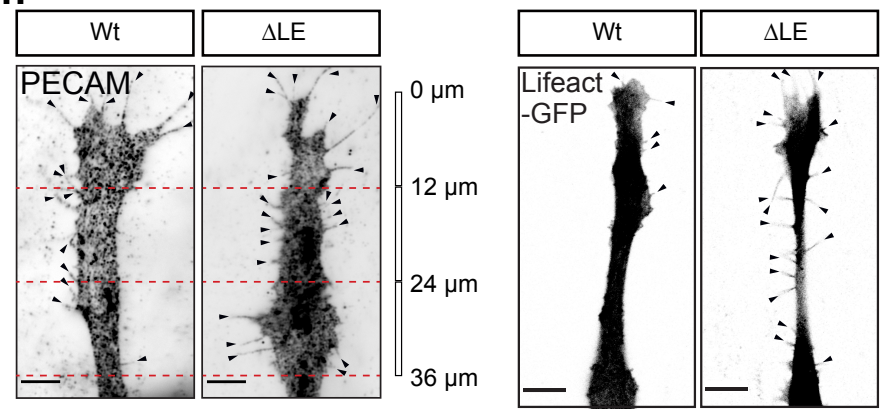

i
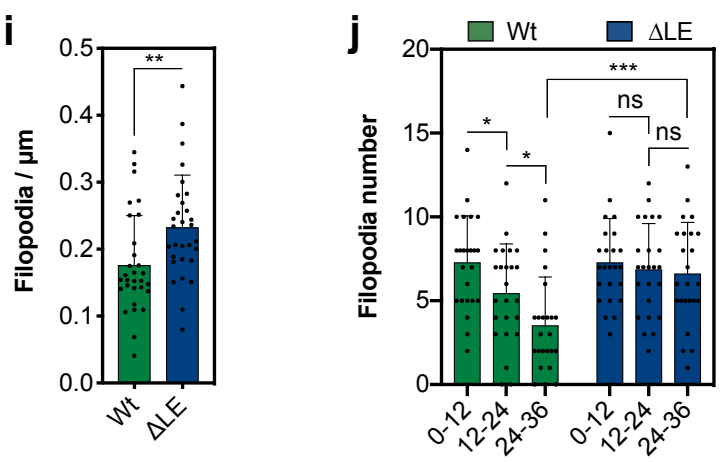

Distance to tip ( $\mu \mathrm{m}$ )

k
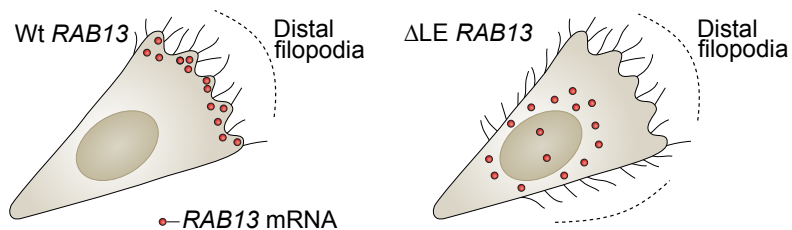

Ectopic proximal filopodia 
bioRxiv preprint doi: https://doi.org/10.1101/374850; this version posted August 11,2019 . The copyright holder for this preprint (which was not certified by peer review) is the author/funder. All rights reserved. No reuse allowed without permission.

Figure 3
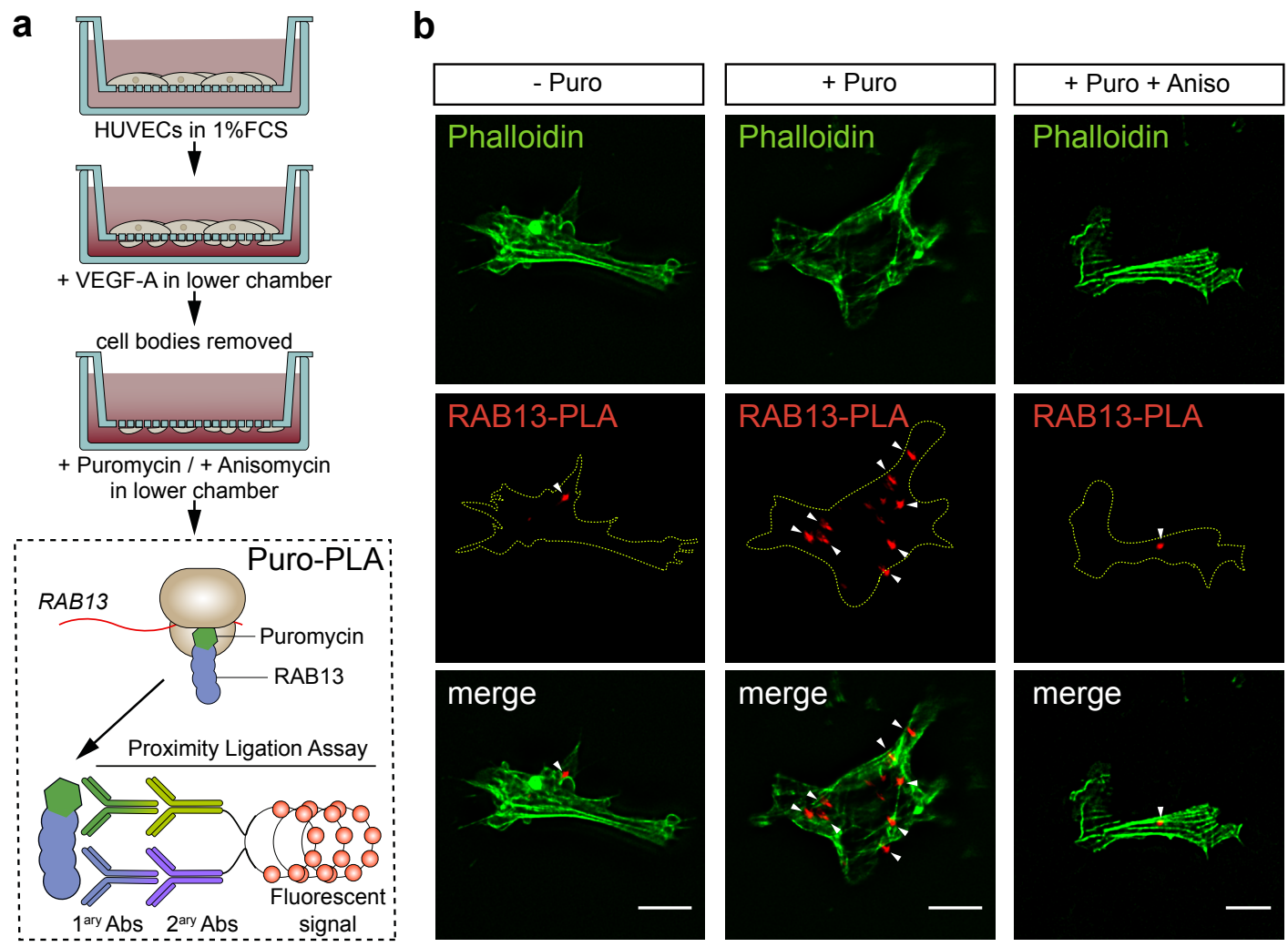

c

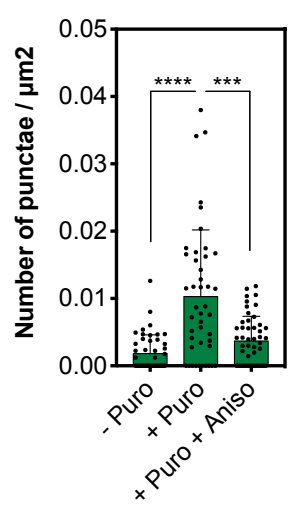

d

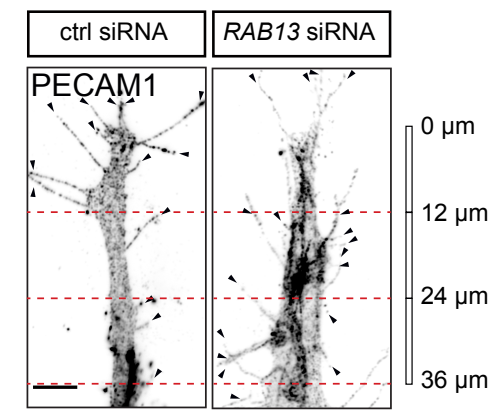

$\mathbf{e}$

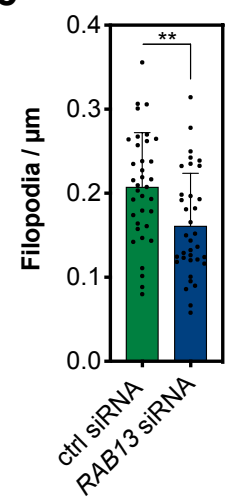

f

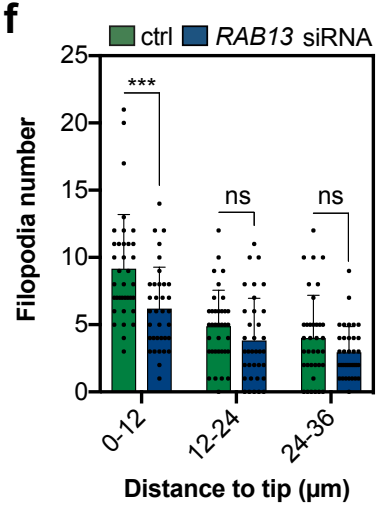

g

RAB13 local translation

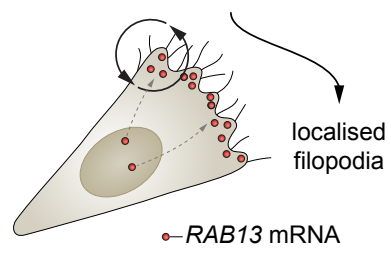


bioRxiv preprint doi: https://doi.org/10.1101/374850; this version posted August 11, 2019. The copyright holder for this preprint (which was

Figure 4

a
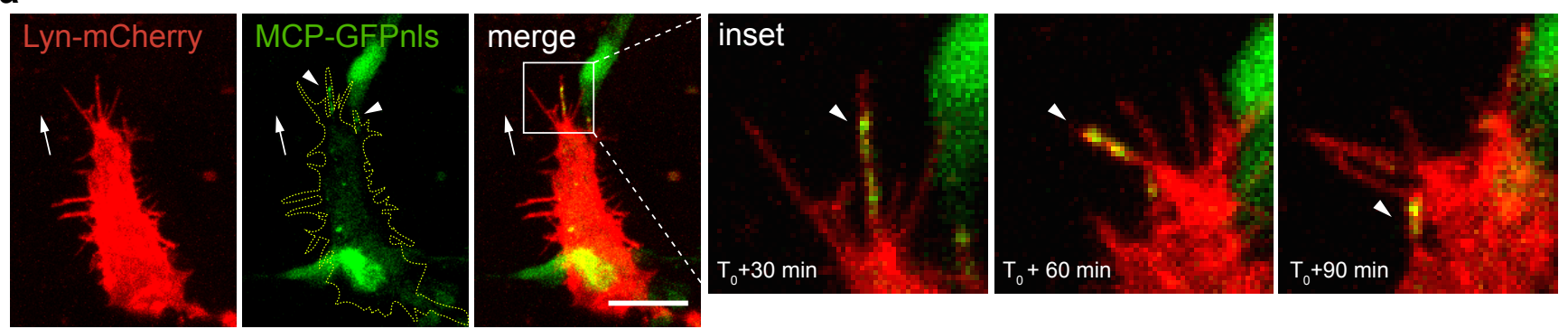

b
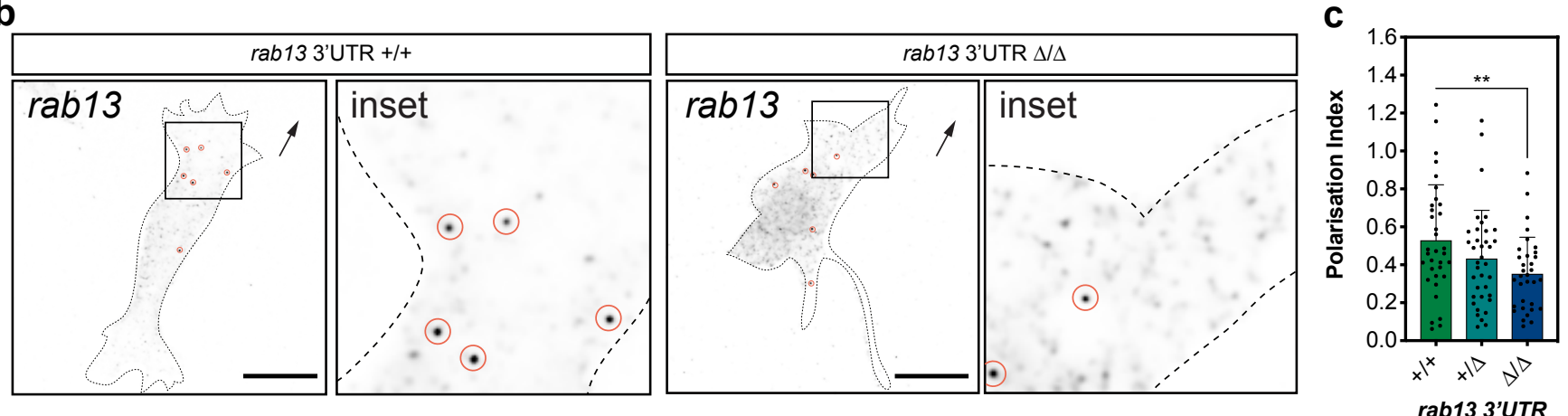

d
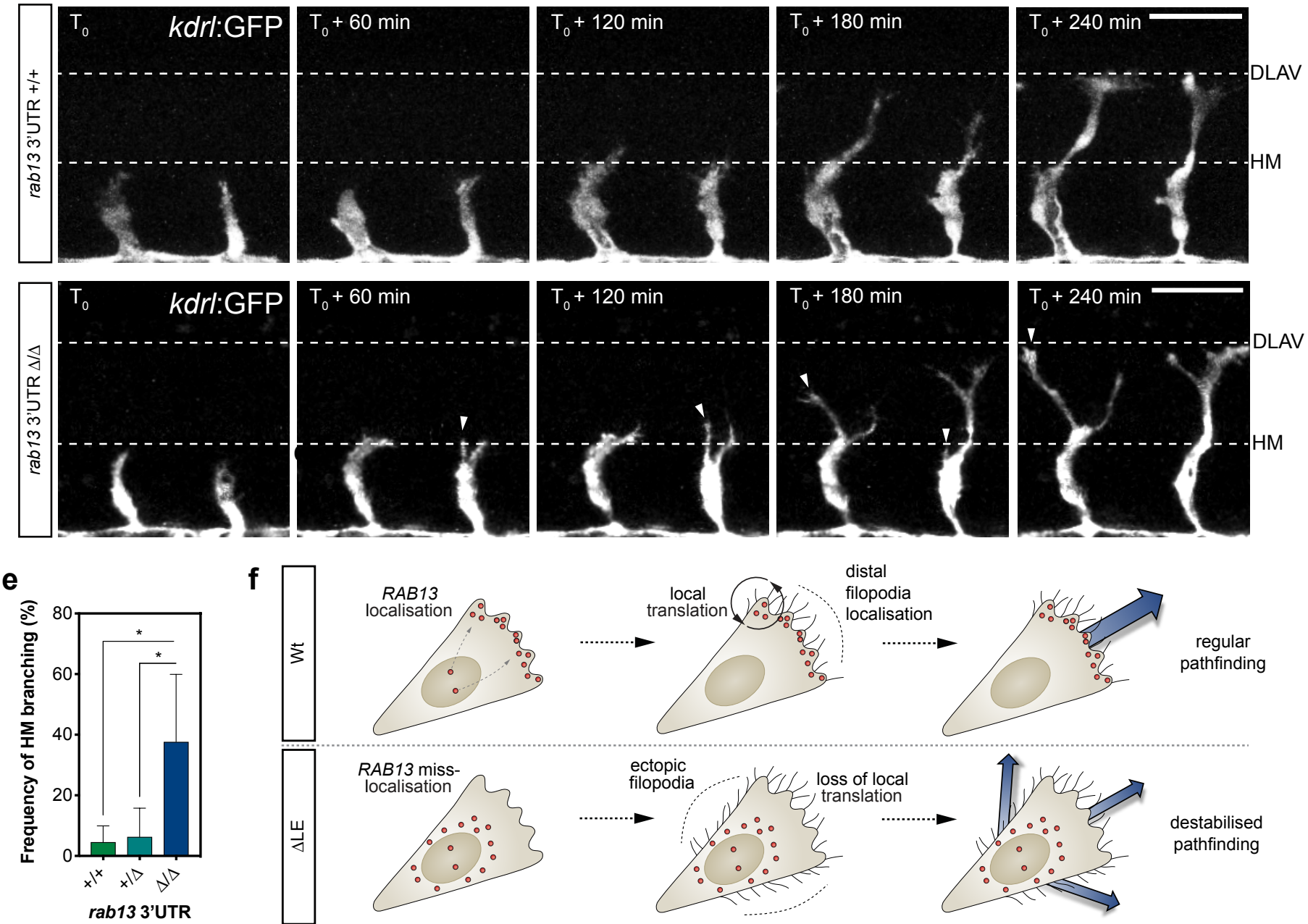

regular pathfinding
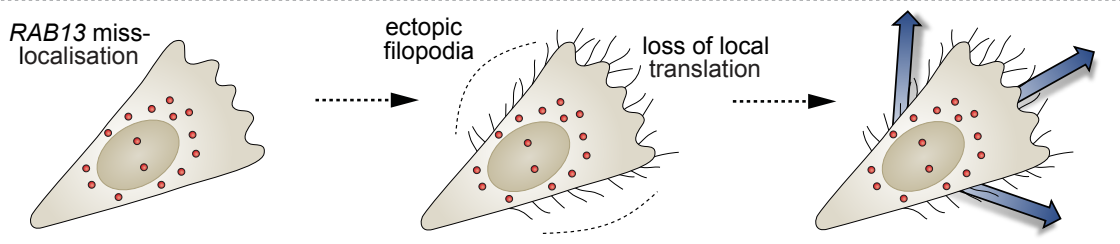

destabilised pathfinding 